\title{
Web et participation politique : quelles promesses et quels pièges?
}

Web and political Participation: what Promises and what Pitfalls?

\section{Peter Dahlgren}

Traducteur : Jean-François Nominé et Richard Nickinson

\section{OpenEdition Journals}

Édition électronique

URL : https://journals.openedition.org/questionsdecommunication/6545

DOI : 10.4000/questionsdecommunication.6545

ISSN : 2259-890

\section{Éditeur}

Presses universitaires de Lorraine

\section{Édition imprimée}

Date de publication : 1 septembre 2012

Pagination : 13-24

ISBN : 978-2-8143-0120-7

ISSN : 1633-5961

\section{Référence électronique}

Peter Dahlgren, «Web et participation politique : quelles promesses et quels pièges ? », Questions de communication [En ligne], 21 | 2012, mis en ligne le 01 septembre 2014, consulté le 27 octobre 2022.

URL : http://journals.openedition.org/questionsdecommunication/6545; DOI : https://doi.org/

10.4000/questionsdecommunication.6545

\section{(c) (i) (9)}

Creative Commons - Attribution - Pas d'Utilisation Commerciale - Pas de Modification 4.0 International - CC BY-NC-ND 4.0

https://creativecommons.org/licenses/by-nc-nd/4.0/ 
PETER DAHLGREN

Media and communication studies

Université de Lund, Suède

Peter.Dahlgren@soc.lu.se

\section{WEB ET PARTICIPATION POLITIQUE : QUELLES PROMESSES ET QUELS PIÈGES ?}

Résumé. - Ici, la principale préoccupation est le rôle d'internet dans la participation des citoyens à la démocratie. En particulier, ce qui concerne le web en tant qu'environnement de plus en plus intégré au quotidien. II s'agit d'abord de situer le web dans le contexte troublé de la démocratie contemporaine. Ensuite, sont retracés plusieurs points clés du débat opposant optimistes et pessimistes au sujet de ce que le web peut faire à la démocratie, avant que ne soit pointé un certain nombre de caractères relatifs à l'environnement web et à la culture des médias, le tout en lien avec thème de la participation. Quant à la dernière section, elle tente de revenir sur la logique de réseau et sa signification positive en termes de participation, offre à cet égard deux tendances problématiques entravant ce potentiel. La discussion finale accentue le caractère ambivalent de rôle démocratique du web, et l'importance de l'analyser dans des contextes spécifiques.

Mots clés. - Internet et démocratie, participation, engagement civique, usage du web. 
$\mathrm{P}$ endant la décennie qui s'est écoulée, je me suis préoccupé de ce problème d'ordre général : la relation entre l'internet et la démocratie. Plus précisément, j'ai étudié comment et à quel point les médias numériques facilitent la participation à la vie politique, comment ils favorisent l'engagement citoyen dans la vie publique. Comme on peut s'y attendre - et c'est une chance - je ne suis pas le seul à m'être lancé dans cette entreprise, et j'ai pu profiter d'amples retours de la part de collègues tandis que j'ai pu lire de nombreux travaux. À ce stade, on peut dire que le sujet « internet et démocratie » constitue en quelque sorte un sous-domaine interdisciplinaire spécialisé qui intéresse quantité de chercheurs venus des études médiatiques, de la communication politique, de la sociologie politique, voire des études culturelles.

Bien entendu, en raison de cette activité de recherche provenant d'horizons variés du monde académique et, dans certains cas, à partir de postulats différents, il ne se dessine pas un paysage sur ce sujet qui soit unique et unifié. En particulier, différentes conceptions de la politique et des représentations variables de la démocratie alimentent ces différences d'appréciation de la situation. Dans ce qui suit, je restituerai mon cheminement personnel, proposerai un panorama synthétique tout en intégrant certains travaux significatifs produits par d'autres chercheurs. Si la participation citoyenne, sous quelque forme qu'elle prenne, peut se concevoir comme une institution, nous devons prendre en compte les contingences de cette institution, que ce soient les circonstances immédiates et les modes de vies quotidiens au niveau individuel des personnes ou les configurations des structures sociales à un niveau plus large. C'est pourquoi je souligne l'importance des conditions socioculturelles modernes récentes pour pouvoir comprendre la démocratie et la participation et, dans ce contexte, la composante médiatique au premier chef. Toutefois, comme je le préconise, nous devons être modestes dans nos attentes quant à ce que le web peut apporter à la démocratie.

J'ai publié assez régulièrement sur ce sujet. Une de mes contributions a été publiée en fait dans la présente revue (Dahlgren 2003a) et on en trouvera une autre en lien avec celle-ci dans une autre publication (Dahlgren, 2003b). J'ai également rendu compte de plusieurs écoles de pensée dans un ouvrage (Dahlgren, 2009) et dans deux anthologies plus spécialement consacrées aux citoyens jeunes (Dahlgren, 2007 ; Olsson, Dahlgren, 20l0). Un ouvrage moins volumineux, également sur les jeunes, est tout récemment paru en français (Dahlgren, 20 I2). Dans la présente contribution, je vais situer le web par rapport à un contexte de difficultés auxquelles la démocratie est confrontée. À partir de là, dans la deuxième partie, je passerai brièvement en revue certains des éléments centraux qui départagent « optimistes » et « pessimistes » dans leurs débats par rapport à ce que le web peut apporter à la démocratie. Dans la troisième partie, je mettrai en lumière un certain nombre d'attributs propres à l'environnement du web et sa culture médiatique, en les reliant au thème de la participation. La quatrième partie étudiera la logique en réseau de l'internet et son importance 
positive pour la démocratie, et offrira aussi deux évolutions problématiques qui grèvent ce potentiel. La discussion en conclusion mettra l'accent sur la nature ambivalente du rôle démocratique du web.

\section{Les difficultés de la démocratie}

Depuis son apparition en tant que phénomène de masse au milieu des années 90 , l'internet est entré dans les travaux de recherche et dans les débats en cours sur la démocratie. Cette attention s'est intensifiée au fur et à mesure que ses dilemmes se sont approfondis. Sa vitalité et sa survie elle-même ne peuvent être considérées comme des évidences. II s'agit d'un projet historique, traversé par des contestations entre les forces qui cherchent à la limiter de diverses façons et celles qui tentent de la développer et de l'approfondir, particulièrement en accroissant la participation des citoyens. Dans les démocraties occidentales contemporaines, la politique de partis semble stagner, n'être que réactive, terne, et nombre de citoyens estiment que l'on ne leur propose pas vraiment de choix. Pour ce qui est de la participation politique, nous avons pu constater une baisse régulière de la participation aux scrutins, de l'engagement dans les partis, et même dans les actions de la société civile. On constate une montée du scepticisme, de la frustration et, disons-le, du cynisme envers la classe politique.

Toutefois, cette tirade sur le déclin de la participation politique se voit contrée par d'autres tendances, pour la plupart hors du champ traditionnel de la politique partisane. Nous voyons émerger une extrême droite très active politiquement dans beaucoup de pays d'Europe, des mouvements qui ne mobilisent pas seulement contre l'immigration, mais expriment aussi les frustrations et le sentiment d'impuissance vécus par un grand nombre de personnes à l'extrémité la plus basse de la hiérarchie socio-économique. En revanche, on constate le retour d'un engagement à gauche, souvent sous la forme de mouvements sociaux progressistes, non traditionnels, qui s'en prennent à la globalisation néolibérale et à l'inégalité socio-économique. Si les effectifs sont difficiles à mesurer, il faut reconnaître, cependant, que ces formes de participation coïncident avec des minorités des segments statistiques du corps civique.

Au mieux, la démocratie apparaît, tout au plus de manière inégale, suite à des luttes politiques. II est rare qu'elle tombe comme un cadeau fait au peuple par les cercles au pouvoir, comme le rappellent les événements du Printemps arabe de 20 I I. Aujourd'hui, les spécialistes universitaires, les journalistes, le personnel politique et les citoyens se demandent si la qualité démocratique de leurs sociétés peut être maintenue et comment. Mais ces personnalités se demandent également de quelles manières on peut traiter nos déficits démocratiques. À cet égard, l'engagement politique représente un thème majeur. En effet, sans un niveau minimum d'engagement de la part de ses citoyens, la démocratie perd sa légitimité, et cesse de fonctionner de manière authentique. Vu sous l'angle de 
l'environnement web, la question qui émerge est celle-ci : dans quelle mesure et comment les médias numériques contribuent-ils à faciliter la participation ?

\section{Web et démocratie : I'ambivalence}

Commençons par examiner l'usage étendu du réseau et de ces technologies accessoires, comme la téléphonie mobile, dans la vie quotidienne. Une proportion croissante de personnes passe de plus en plus de temps à satisfaire un éventail de besoins dans cet espace omniprésent qu'est le web. Le réseau n'est pas simplement un emplacement que l'on visite occasionnellement pour y chercher quelque chose de particulier, il devient de plus en plus le terreau central de la vie quotidienne individuelle. Les jeunes sont particulièrement consommateurs de ses outils, non seulement pour envoyer des messages parlés ou écrits, mais aussi pour télécharger, remixer, relier et partager des contenus divers, et ce, avec des compétences d'une complexité de plus en plus accrue. Que ce soit pour interagir sur des réseaux sociaux avec des amis, converser sur des blogs, butiner de la musique ou des informations, faire ses courses ou trouver un partenaire, le web est le principal lien avec le monde. Le réseau se mêle de la manière la plus intime à la vie individuelle extérieure à ce dernier, tout comme au fonctionnement des groupes, des organisations et des institutions. Si le web offre un environnement important pour la participation sociétale, il n'est guère - en particulier chez les plus jeunes - ressenti comme radicalement à part de leur vie en général. Toutefois, sur le plan sociologique, on peut avancer (voir supra) que certaines fonctions sociales nécessitent aussi des éléments de rencontres in vivo, en face à face, pour pouvoir être reconnues. La démocratie en fait partie.

Cette transformation spectaculaire du paysage médiatique comme de l'univers social entraîne évidemment des conséquences pour la démocratie. Sur ce point, on voit la visibilité des débats s'accélérer, avec ces champs de force de l'optimisme et de pessimisme très proches de nous. Si certains observateurs (Benkler, 2006 ; Castells, 20l0) soulignent l'effet globalement positif du web sur la démocratie, des auteurs (Morozov, 20 I I) avancent que cet argument est surfait, et que non seulement les technologies de l'internet ne contribuent pas à démocratiser le monde mais qu'elles sont aussi utilisées par des régimes autoritaires pour contrôler leurs citoyens et museler les oppositions. Vu sous l'angle cognitif, Nicholas Carr (2010) avance l'idée selon laquelle les médias numériques réduisent la capacité à penser, lire et se souvenir, rendant de fait problématiques les fondements mêmes de la civilisation. Si de nombreux analystes partagent l'idée de Cass R. Sunstein (2008) voulant que la « sagesse des foules » (telle qu'elle se concrétiserait dans Wikipédia et sur la blogosphère) produit démocratiquement des formes de connaissances nouvelles et améliorées, d'autres comme Andrew Keen (2008) pointent du doigt les dangers du web 2,0 participatif et affirment qu'il érode les valeurs, les normes et la créativité. 
Mais des observateurs soulignent également que l'utilisation du web à des fins politiques est largement moins prioritaire que la consommation, les loisirs, les liens sociaux... Par exemple, Mathew Hindman (2009) estime que seul 0, 10 \% du trafic s'oriente vers les sites politiques (contre $10 \%$ vers les sites pornographiques). Pour la majorité des personnes, les problèmes politiques ne sont pas le sujet majeur de préoccupation, et si le web est un outil impressionnant, il ne suffit pas à lui seul à mobiliser des citoyens qui ne seraient pas enclins à s'engager.

II serait trop simple d'écarter ces analyses à tendance sceptique. En même temps, la recherche a continué à souligner les potentialités qu'offre l'internet pour élargir et approfondir l'engagement démocratique. Si la politique continue à représenter un usage limité du réseau, son vaste univers de communication facilite son apparition dans les échanges en ligne. On peut même dire qu'elle y « fait irruption », spécialement sous ses nouvelles formes post-partisanes qui se manifestent actuellement. L'environnement du web offre un large éventail de formes de participation politique qui adoptent différentes formules : sites web pour groupes d'activistes, forums de discussions et de débats, documentaires sur des manifestations et affrontements politiques mis en ligne sur YouTube. Qui aurait pensé, à leurs débuts, que Facebook et Twitter deviendraient d'importantes institutions de la scène publique, jouant un rôle dans les débats et la formation des opinions? Certes, si l'incertitude demeure, nous pouvons toutefois mettre en lumière un certain nombre de caractéristiques relatives au rôle du réseau dans la démocratie. Mais qu'en est-il de quelques thèmes essentiels relatifs à la participation démocratique via le web.

\section{La participation et l'environnement dynamique du web}

Dans le contexte démocratique, traiter de la participation peut conduire à des problématiques complexes (pour une approche théorique, voir Carpentier, 20 I I). Par exemple, il peut être ardu de définir pleinement sur le plan conceptuel ou empirique à quel point les gens sont intégrés « aux médias » ou, plus largement, à la société « via les médias », car les univers médiatiques et sociaux s'enchevêtrent bien au-delà d'eux-mêmes. La fonction médiatrice des premiers nous conduit à être liés à des réalités sociales qui dépassent l'immédiateté géographique et temporelle (l'ici et/ou le maintenant). L'intensité avec laquelle les personnes valorisent leur expérience des médias par rapport à celui qui les met en relation, restera une question ouverte, mais l'éclairage qui sera donné sur les motivations et les intentions des participants sera indicatif de ce qu'elles perçoivent comme essentiel.

De façon plus significative, nous pouvons nous interroger sur ce qui importe dans la participation dite « politique », particulièrement dans une situation où 
la politique subit, comme aujourd'hui des changements importants comme le soulignent de nombreux auteurs. Les jeunes citoyens peuvent développer le sentiment que le monde politique conventionnel ne les attire pas, ce qui ne veut pas pour autant dire qu'ils se détournent des questions politiques. Ceux qui s'engagent recherchent souvent des points d'entrées dans les questions de société, tout comme de nouvelles formes de pratiques et d'expression politiques, et c'est à ce niveau que le réseau trouve toute sa raison d'être (Dahlgren, 2007 ; Olsson, Dahlgren, 2010 ; Bennett, 2007 ; Buckinghan, 2007). Leur engagement est davantage motivé par des préoccupations normatives personnelles (souvent liées au développement de leur identité) que par les idéologies traditionnelles, leur intérêt allant plus vers des questions précises plutôt que sur des problèmes sociaux plus larges. S'il y a un certain danger à s'en tenir à une vision trop limitée de l'action politique, la contestation dans ce domaine (Mouffe, 2005) peut surgir n'importe quand et n'importe où et prendre des formes d'expression toujours plus nouvelles.

La culture populaire n'est pas la dernière à se faire l'écho d'une dimension politique et tend à se superposer sur la scène politique (voir Street, 1997 ; van Zoonen, 2006 ; Riegert, 2007). Elle peut exprimer des valeurs démocratiques importantes par son côté accessible et convivial, elle engage à la participation, ouvre facilement les portes vers des communautés symboliques, vers un monde où l'on dépasse son appartenance individuelle. À certains moments, ceci peut préparer à une participation citoyenne en offrant ce que Joke Hermes (2005) désigne comme une « citoyenneté culturelle ». Sur le net, la culture populaire devient d'autant plus participative et interactive qu'elle propose des sujets et des moyens qui encouragent à s'engager envers de nombreuses questions comme nos modes de vie et la société que nous voulons. Ce genre de débats permet d'aborder et d'élaborer différentes sortes de positions associées à des valeurs, des normes et des identités qui se trouvent contestées dans les turbulences de notre environnement socioculturel post-moderne, même dans des moments où sont actualisés des conflits où une distinction d'identités entre des « nous » et des « eux » est possible. II devient difficile de tracer des lignes de démarcation, mais sur le plan conceptuel, on peut dire que ce terrain se situe en un endroit que Bernard Miège (20I0) dénomme la scène publique sociétale, qu'il a distinguée de la scène publique plus officielle.

II en va de même du journalisme, domaine dont les frontières sont devenues moins nettes. Le monde occidental connaît une « crise du journalisme » à grande échelle, question qui dépasse mon champ d'intervention ici (toutefois, pour une vision générale de la situation aux États-Unis, voir les rapports annuels disponibles sur stateofthemedia.org). Ce qui m'intéresse c'est le journalisme comme domaine en évolution et constitutif d'un terrain pour la participation citoyenne, car de nombreux citoyens considèrent le journalisme comme leur mode de participation. Ce que nous voyons apparaître est un univers hétérogène, formé en particulier par la blogosphère, mais aussi de médias sociaux comme Facebook 
et Twitter, de productions individuelles et de groupes, y compris les actions entreprises par des mouvements sociaux et militants de tous bords : des groupes politiques et religieux, les promoteurs de certains styles de vie, de certains passetemps, et bien d'autres cas encore. Voilà un bouillon de culture journalistique extrêmement vivant et chaotique, où faits et opinions interviennent, des débats, des ragots, des absurdités, de la désinformation, du perspicace, du trompeur, du poétique, le tout mélangé. Souvent, il brouille les frontières traditionnelles entre la sphère publique et la vie privée, parfois à bon escient, d'autres fois moins. Toutefois, il y a là bien des aspects encourageants sur le plan démocratique, et le ton parfois dithyrambique de bien des propos sur le journalisme participatif se justifie souvent.

Cependant, le sociologue se doit de garder la tête froide dans l'étude de ces évolutions, car elles suscitent tant de questions (voir Papacharissi, 2009 ; Rosenberry, Burston, 2010 ; Tunney, Monaghan, 2010). Ainsi une bonne part du journalisme citoyen fonctionne-t-il en symbiose avec les supports classiques, quand bien même les commente-t-il et les conteste-t-il. Dans leur étude sur le journalisme sur la blogosphère, Vincent Campbell, Rachel Gibson, Barrie Gunter et Maria Touri (2010) ont constaté que les blogueurs qui ne sont pas des journalistes professionnels ne produisent que très rarement des informations originales. Plus fondamentalement, quand les frontières du journalisme se brouillent, les normes de sa pratique et ses critères d'évaluation deviennent périlleuses à leur tour. Pour le journalisme participatif - que bien souvent les convictions d'une démocratie citoyenne alimentent davantage que des valeurs professionnelles traditionnelles -, cela veut dire que des références usées par le temps comme la véracité, la transparence, l'équité et la responsabilité posent problème. La question classique « à qui peut-on faire confiance » se pose avec encore plus d'intensité.

La culture médiatique semble s'éloigner encore plus des idéaux que comportent les représentations traditionnelles de l'espace public rationnel, alors qu'en même temps, elle engendre de nouvelles pratiques et des modes d'expression que nous devons prendre en compte. C'est ainsi que Leah A. Lievrouw (201 I :214) décrit pertinemment cette situation : « La culture médiatique à l'ère du numérique est devenue plus individuelle, sceptique, ironique, périssable, idiosyncratique, collaborative, et presque incroyablement, diversifiée... ». En réalité, ce que cette auteure restitue ici constitue une partie de ces textures définitives que prend la situation post-moderne, où s'entrecroisent des courants dans les relations de pouvoir et leurs sensibilités particulières, ainsi que leurs tensions structurelles. C'est à l'aide de ces toiles de fond historiques que nous devons comprendre la participation, la politique et la citoyenneté médiatique. Cette analyse souligne l'interaction entre l'affordance propre aux technologies de la communication et les pratiques d'utilisation. Au niveau de cette interface, les gens adaptent, réinventent, réorganisent ou reconstruisent les technologies des médias autant qu'il est nécessaire pour correspondre à leurs différentes objectifs ou intérêts. À 
mesure qu'ils innovent, les utilisateurs associent anciennes et nouvelles techniques, ou adaptent différemment des combinaisons technologiques connues. Les nouveaux médias sont recombinants, le résultat d'une hybridation de technologies existantes avec des techniques nouvelles (Lievrouw, 201 I :216).

Plus concrètement, cette approche permet de comprendre la pertinence des pratiques citoyennes numériques dans ces ensembles participatifs. En effet, ce que les gens font bel et bien grâce au web prend de l'importance pour la participation, et leurs actions sont sans cesse dans la nouveauté. À leur tour, ces pratiques engendrent une évolution progressive des espaces publics. Les pratiques s'instaurent en tant que moyens où peut puiser la nouvelle participation. Si le côté strict de la théorie discursive habermassienne est fréquemment marginalisé, on peut cependant avancer que ces évolutions produisent des modes de communication contemporains qui sont plus au diapason avec les tempéraments culturels en vogue, et donc facilitent d'autant plus l'expression et la formation de l'opinion publique. Pourtant, à ces postulats s'ajoute sans aucun doute à ce que l'on pourrait appeler une « volatilité communicationnelle » qui recèle autant de promesses que de pièges.

\section{Réseaux, chambres d'écho et solosphères}

Ici, en changeant de focale, nous pouvons dire que la participation politique, en tant que phénomène collectif plus que purement individuel, nécessite des réseaux sociaux et contribue à la vitalité de l'espace public. En sciences sociales, l'idée des réseaux sociaux ne date pas d'hier ; elle a été le centre d'intérêt de nombreux travaux de recherche dans les décennies passées. Dans le contexte de l'internet, depuis les années 90, les travaux de Manuel Castells (2000) jouent bien entendu un rôle important (voir aussi Cardosa, 2006). Ce dernier avance que l'« espace des flux » de la société en réseaux émergente remplace l'organisation spatiale qui donnait forme à notre expérience, l'« espace des lieux », et que cette logique transparaît dans de nombreux secteurs, y compris l'architecture urbaine. L'internet représente bien sûr la part la plus emblématique de cette évolution. Le lieu en tant que tel n'a pas été éradiqué, et nous continuons à vivre nos existences par rapport à des lieux géographiques précis. Mais la fonctionnalité des relations sociales fondées et appuyées sur la médiation des réseaux devient de plus en plus centrale. Dans ses travaux récents, Manuel Castells (2010) propose un renouvellement du concept, notamment compte tenu d'une exploitation accrue de sa part de la littérature scientifique issue des études médiatiques et de la communication.

Les réseaux se caractérisent souvent par le côté libre des liens sociaux, relativement faciles à créer et entendus comme ayant des limites définies en termes d'obligation. Ce type de liens est un avantage pour la culture démocratique, étant donné qu'ils reproduisent les relations entre citoyens dans l'espace public, 
c'est-à-dire une coopération fondée sur la confiance dans le cadre d'objectifs communs, mais sans les exigences des relations primaires. L'idée que les réseaux soient une morphologie sociale dominante gagne du terrain en ce qu'elle représente un point de vue utile pour comprendre le monde moderne, et il importe de souligner les avantages sociaux des réseaux. Ils permettent d'éviter les handicaps créés par l'isolement, favorisent le développement social (et politique), forgent les identités collectives, inspirent et permettent d'imaginer des solutions de remplacement. Nancy K. Baym (2010) analyse en détail la manière dont les médias numériques - par leur influence et leurs capacités interactives, les modalités de leurs signaux sociaux, leur structure temporelle, leur mobilité et autres caractéristiques - contribuent à faciliter les relations sociales. Voilà un trait significatif en soi, mais j'inclinerais aussi à souligner que cette lubrification du social est également essentielle pour que le politique fasse son apparition dans les réseaux sociaux. Bref, on pourrait dire que les médias numériques favorisent une sensation d'autonomie au niveau subjectif, un meilleur sens des responsabilités dans la communication horizontale en réseaux.

Cependant, cette logique est mise en cause sur deux fronts au moins. Pour commencer, il y a cette notion connue des chambres d'écho, et ensuite ce que j'appelle les « sphères solo ». Très tôt, les commentateurs ont élaboré l'expression de « chambres d'écho » pour désigner la tendance des personnes à se regrouper à l'intérieur de réseaux par communautés d'opinions. II s'agit d'un schéma de comportement humain compréhensible : on évite les confits et renforce ses visions du monde et ses valeurs. Cela prend tout son sens sur le plan social. Mais si l'on transpose cette tendance dans le contexte des réseaux, on constate un danger pour la démocratie, car ces mini-espaces publics tendent à isoler leurs membres des plus grands courants de débats qui animent le champ sociopolitique. De plus, chez leurs participants, ils réduisent leur expérience à un niveau limité de confrontation à d'autres points de vue ainsi que leur compétence à prendre part à des conflits d'idées. La dimension du dialogue dans l'espace public s'amenuise quand des groupes politiques se jettent des invectives à la figure, sans jamais s'engager dans un débat ou développer une capacité pour la délibération citoyenne. Cette évolution se renforce avec les médias sociaux où la logique absolue est le « j'aime ça » : autrement dit, on clique pour les gens qu'on « aime », c'est-à-dire qui sont comme soi-même. Les différences sont exfiltrées, phénomène dommageable pour l'intelligence comme pour la démocratie dans son fonctionnement, construite pour la résolution des conflits par la discussion.

J'emploie le terme de solosphère pour décrire un processus individualisé d'engagement politique sur le web. Dans l'univers postmoderne, on trouve en ligne des masses de présentations personnelles, un « travail d'identité » induisant une visibilité personnelle, de l'autopromotion et une révélation de sa petite personne. Cela présente un certain nombre d'avantages, mais soulève aussi des problèmes. Des médias sociaux comme Facebook sont devenus des sites fortement interactifs qui ne visent pas nécessairement les rencontres en 
dehors du réseau, un modèle qui commence à se faire sentir dans la participation politique. Pour le long terme, il faut qu'existent des liens entre les vécus en ligne et hors ligne. Zizi Papacharissi (2010) énonce que si les citoyens versés dans le numérique sont certes plus compétents et critiques sous bien des aspects, toutefois, ils sont de plus en plus éloignés des habitudes collectives, sociales et citoyennes du passé.

C'est pourquoi aujourd'hui une bonne partie du comportement citoyen tire ses origines des milieux privés, et elle suggère que ce phénomène donne naissance à de nouveaux « dialectes citoyens ». On peut considérer solosphères comme un habitus historiquement nouveau de la participation en ligne, un autre mode de responsabilité citoyenne. À partir de ses compartiments sur le réseau, souvent sur mobile, de son espace personnalisé, la personne est impliquée dans un large éventail d'environnements dans le monde extérieur. Dans un certain sens, ce phénomène peut signer un retrait dans un environnement que la plupart des gens a l'impression de mieux maîtriser. Une « socialité en réseau » mais néanmoins privée apparait. Dans la mesure où cela serait vrai, cela peut se comprendre tout en introduisant une urgence historiquement neuve envers la participation, ce qui, à son tour, peut préfigurer une forme historiquement neuve du système démocratique.

\section{Conclusion}

La question apparemment simple sur le fait que et la manière dont le web facilite la participation chez les citoyens ne suscite pas de réponse univoque, une ambiguïté demeure. À ce niveau, il n'existe pas de lien direct de « cause à effet », ceux qui pensent que « le réseau va sauver la démocratie » sont peu nombreux. Toutefois, si la recherche a été prudente en général, dans le sens où il n'est pas été offert de belles solutions technologiques pour les difficultés de la démocratie, elle a cependant continué de souligner l'idée que l'internet peut faire la différence. En contribuant aux transformations massives de la société contemporaine à tous les niveaux, il a aussi changé spectaculairement les emplacements et l'infrastructure de l'espace public de quantité de manières. II a rendu accessibles d'immenses masses d'informations, encouragé la décentralisation et la diversité, facilité la communication interactive et individuelle, ainsi que favorisé de nouvelles pratiques citoyennes - tout en fournissant, notamment, un espace de communication citoyenne pratiquement illimité à qui le souhaite. Ce faisant, l'environnement web a redéfini les lieux et le caractère de la communication politique. II paraît justifié de caresser de modestes espoirs.

\section{Traduit de l'anglais par Jean-François Nominé (Inist, Nancy)} et Richard Nickinson (Inist, Nancy) 


\section{Références}

Baym N. K., 20 I 0, Personal Connections in the Digital Age, Cambridge, Polity Press.

Benkler Y., 2006, The Wealth of Networks : How Social Production Transforms Markets and Freedom, New Haven, CT :Yale University Press.

Bennett W. L., ed., 2007, Civic Life Online : Learning How Digital Media Can Engage Youth, Cambridge, MA MIT Press. Accès : http://mitpress.mit.edu/catalog/browse/browse. asp?btype $=6 \&$ serid $=170$.

Buckingham D., ed., 2007, Youth, Identity and the Digital Media, Cambridge, MA : MIT Press. Accès : http://mitpress.mit.edu/catalog/browse/browse.asp?btype=6\&;serid= 170.

Campbell V., Gibson R., Barrie G., Touri M., 2010, « News blogs, mainstream news and news agendas », pp. 31-47, in :Tunney S., Monaghan G., eds, Web Journalism ://A New Form of Citizenship? Brighton, Sussex Academic Press.

Cardosa G., 2006, The Media in the Network Society : Browsing, News, Filters and Citizenship, Lisbon, Centre for Research and Studies in Sociology.

Carpentier N., 20I I, Media and Participation: A Site of Ideological-Democratic Struggle, Bristol, Intellect Publishers.

Carr N., 2010, The Shallows: How the Internet is Changing the Way We Think, Read and Remember, London, Atlantic Books.

Castells M., 2000, The Rise of the Network Society, $2^{\text {nd }}$ ed., Oxford, Blackwell.

— 2010, Communication Power, Oxford, Oxford University Press.

Dahlgren P., 2003a, « À la recherche d'un public parlant : les médias et la démocratie délibérative », pp. 29|-312, in : Cefaï D, Pasquier D., éds, Les sens du public, Paris, Presse universitaires de France.

— 2003b, « La reconfiguration de la culture civique », Questions de communications, 3, pp. $|5|-168$.

— ed., 2007, Young Citizens and New Media : Learning for Democratic Participation, New York, Routledge.

- 2009, Media and Political Engagement : Citizens, Communication, and Democracy, New York, Cambridge University Press.

- 2012, «L'environnement du web, les cultures citoyennes et les jeunes », Jeunes et médias, 3, printemps.

Hermes J., 2005, Re-reading Popular Culture, Oxford, Blackwell.

Hindman M., 2009, The Myth of Digital Democracy, Princeton, NJ Princeton University Press.

Keen A., 2008, The Cult of the Amateur, New York, Doubleday.

Lessig L., 2006, Code :Version 2.0, New York, Basic Books.

Lievrouw L. A., 201 I, Alternative and Activist New Media, Cambridge, Polity Press.

Miège B., 2010, L'Espace public contemporain, Grenoble, Presses universitaires de Grenoble. 
Morozov E., 201 I, The Net Delusion : the Dark Side of Internet Freedom, New York, Public Affairs Books.

Mouffe Ch., 2005, On the Political, London, Verso.

Olsson T., Dahlgren P., eds, 20I0, Young People, ICTs and Democracy, Gothenburg, Nordicom.

Papacharissi Z., 2010, A Private Sphere: Democracy in a Digital Age, Cambridge, Polity Press.

— 2009, Journalism and Citizenship : New Agendas in Communication. London, Routledge.

Reigert K., ed., 2007, Politicotainment :Televison's Take on the Real, New York, Peter Lang Publishers.

Rosenberry J., Burston St. J., eds, 20 I0, Public Journalism 2.0: The promise and Reality of a Citizen-Engaged Press, London, Routledge.

Strangelove M., 2005, The Empire of Mind : Digital Piracay and the Anti-Capitalist Movement, Toronto, University of Toronto Press.

Street J., 1997, Politics and Popular Culture, Cambridge, Polity Press.

Sunstein C., 2008, Infotopia : How Many Minds Produce Knowledge, New York, Oxford University Press.

Tunney S., Garrett M., 20 I0, eds, Web Journalism ://A New Form of Citizenship ? Brighton, Sussex Academic Press.

Van Zoonen L., 2005, Entertaining the Citizen: When Politics and Popular Culture Converge, Lanham, MD : Rowman \& Littlefield. 